Nancey Murphey, George F.R. Ellis, and Timothy O'Conner, eds., Downward Causation and the Neurobiology of Free Will (Springer, 2009), pp. 187-197.

\title{
11
}

\section{Contemplative Neuroscience as an Approach to Volitional Consciousness}

\author{
Evan Thompson \\ Department of Philosophy \\ University of Toronto \\ 170 St. George Street, 4th floor \\ Toronto, ON M5R 2M8 \\ Canada \\ evan.thompson@utoronto.ca
}

Summary. This chapter presents a methodological approach to volitional consciousness for cognitive neuroscience based on studying the voluntary selfgeneration and self-regulation of mental states in meditation. Called contemplative neuroscience, this approach views attention, awareness, and emotion regulation as flexible and trainable skills, and works with experimental participants who have undergone training in contemplative practices designed to hone these skills. Drawing from research on the dynamical neural correlates of contemplative mental states and theories of large-scale neural coordination dynamics, I argue for the importance of global system causation in brain activity and present an "interventionist" approach to intentional causation.

Key words: volition, consciousness, neurodynamics, neurophenomenology, contemplative neuroscience, meditation.

In this chapter I sketch a methodological approach to volitional consciousness for cognitive neuroscience based on studying the voluntary self-generation and selfregulation of mental states in meditation. Called contemplative neuroscience, this approach views attention, awareness, and emotion regulation as flexible and trainable skills, and works with experimental participants who have undergone extensive training in contemplative practices designed to hone these skills. My discussion here is premised on the following three working assumptions (Lutz et al. 2007; Lutz et al. 2008): 
- Advanced contemplative practitioners can generate new data that would not exist without contemplative mental training. These include various conscious states and processes occurring during contemplative practices as well as longer lasting traits that may be brought about by these practices.

- Advanced contemplative practitioners can reliably reproduce and maintain specific aspects or types of conscious processes. These include focused attention, one-pointed concentration, and various types of meta-cognitive awareness. This ability to stabilize conscious processes makes them easier to investigate experimentally.

- Advanced contemplative practitioners can give precise first-person descriptions of conscious mental states. This information is relevant for refining psychological taxonomies of the full range of conscious states and for interpreting neuroimaging data about conscious processes.

\section{Toward a Neurophenomenology of Volition}

Most cognitive neuroscientists studying consciousness maintain that self-reports of experience provide indispensable evidence about conscious processes (Jack \& Roepstorff 2002). Although scientists often acknowledge the conceptual distinction between phenomenal consciousness (subjective experience) and access consciousness (cognitive access and reportability) (Block 1997), they usually argue that there is no clear scientific criterion for defining a process as conscious apart from its reportability (e.g., Dehaene \& Naccache 2001). Nevertheless, as psychologists have discussed, being able to gain access to experience and report it is a cognitive capacity in its own right. When asked to report and describe our experiences - for example, our experiences of conscious will (Wegner 2002, 2004) - we need to introspect in order to become explicitly aware of our experience. In other words, we must consciously represent to ourselves our experience, thereby engaging metaconsciousness or meta-awareness, a distinct form of meta-cognition (Schooler 2002).

It is well known that self-reports requiring introspection or meta-awareness are subject to various biases, especially when subjects are asked or allowed to report what they take to be the causes of their experiences (Nisbett \& Wilson 1977; see also Hurlbert \& Heavey 2001; Schooler 2002). Yet even when subjects are discouraged from offering reasons or explanations, and are encouraged simply to describe their experiences as carefully as possible, a variety of interrelated difficulties present themselves (Petitmengin 2006; Schooler 2002; Schooler \& Schreiber 2004).

One difficulty has to do with the instability of attention. Our attention tends to jump rapidly from one thing to another. As William James observed, in the case of voluntary attention, it takes considerable effort to sustain attention on a given 
object (James 1985, p. 91). Such mental effort seems especially present when the target of attention happens to be our experiences or mental processes and the type of attention is accordingly endogenous attention requiring top-down cognitive control.

A second difficulty is lack of awareness of experience. Not only does our attention jump around, but we usually have little or no explicit awareness of this attentional instability. Mind-wandering is a familiar case. Engaged in some task such as reading or writing, our attention wanders and we become lost in spontaneously arising thoughts and memories. Although we are conscious and have a variety of experiences with specific contents, we have little or no explicit awareness of those experiences as they occur. If we catch ourselves daydreaming, we then become meta-conscious of our subjective mental activity. In such cases there is a "temporal dissociation" between consciousness and meta-consciousness, "in which the triggering of meta-consciousness causes one to assess aspects of experience that had previously eluded explicit appraisal" (Schooler 2002, p. 340).

A third difficulty is that introspection or meta-awareness can change experience, particularly if the experience is nonverbal and verbalization is required (Schooler 2002). For example, directing attention towards an emotion and trying to verbalize it can change its phenomenal character in a variety of ways (Lambie \& Marcel 2002).

Finally, a related difficulty is the possibility for misrepresentation that gets introduced when one has to represent the contents of consciousness in meta-consciousness. Schooler (2002) calls this sort of dissociation between consciousness and meta-consciousness "translational dissociation": "If meta-consciousness requires re-representing the contents of consciousness, then, as with any recoding process, some information could get lost or become distorted in the translation" (Schooler 2002, p. 342).

As scientists aiming for a better understanding of consciousness, we thus find ourselves in the following situation. On the one hand, self-reports of experience are indispensable and arguably the main source of evidence for the presence of a given conscious process. On the other hand, gaining cognitive access to experience and being able to report and describe it with precision are abilities that present their own challenges and presumably vary across individuals (as readers of novels already know).

Given this situation, we might conjecture that individuals who strive to develop a high degree of intimacy with and control over their own subjective mental processes through contemplative training of attention and meta-awareness can provide more detailed and accurate self-reports about the contents of consciousness (Lutz et al. 2008). This conjecture is one of the working assumptions of contemplative neuroscience (Lutz et al. 2007).

Volitional consciousness provides a potential case study for testing this conjecture. There has been little sustained investigation of the phenomenology of volition (even in the tradition of Phenomenology), particularly of the sorts of volitional experiences philosophers appeal to when they defend one or another account of free will (Nahmias et al. 2004). At the same time, psychologists such as Wegner 
$(2002,2004)$ have concluded that the experience of conscious will as causally efficacious is illusory, though careful attention to the phenomenology of volition and agency does not seem to support this conclusion (Bayne 2006). Clearly, more phenomenological work needs to be done to investigate the experience of volition. Yet how should such investigation proceed?

Nahmias and colleagues propose that we should investigate the "folk phenomenology of free will," suggesting that "reports gathered from laypersons will be minimally tainted by philosophical theory" (Nahmias et al. 2004, p. 172). ${ }^{1}$ They note the need for introspective reports from subjects who are not trained in theoretical debates (unlike the nineteenth- and early twentieth-century Introspectionists). Yet they also realize that obtaining self-reports about the experience of volition encounters difficulties of the sort discussed above:

Our goal is to understand the phenomenology of free will in a way that informs the theoretical debate without tainting the phenomenology itself. But one might argue that, without training, the phenomenology of free will is either too difficult to apprehend or to describe or both. So, even if there is a folk phenomenology of free will, we may be unable to get systematic descriptions of it from folk who have yet to be trained in some relevant way.... For this purpose, it would be helpful for psychologists, perhaps guided by the questions raised in the philosophical debate, to reconsider the basic introspectionist project of gathering first-person reports, even offering some guidance about how to attend to conscious phenomena, while avoiding the introspectionists' tendency to train subjects in the theoretical debates. If the data from subjects' reports can be triangulated with behavioural and neuropsychological data, all the better. (Nahmias et al. 2004, p. 172)

Here we need to distinguish between training in theoretical debates and training in mental skills involving attention to and awareness of conscious processes. In calling for psychologists to offer guidance in how to attend to conscious phenomena, Nahmias and colleagues acknowledge that such attention can be guided and thus trained. They recommend the phenomenological interview, which uses openended questions to guide subjects to describe their experience while directing them away from trying to explain it. Such guidance is a form of training, for subjects learn to attend to their experience without trying to rationalize it or explain its causes (Petitmengin 2006).

This procedure of combining first-person phenomenological investigation, second-person phenomenological interviews, and third-person behavioral and neurophysiological measures is central to the approach known as neurophenomenology (Lutz et al. 2002; Lutz \& Thompson 2003; Varela 1996). Thus, one way to make headway in understanding volitional experience and its relation to the brain is to pursue a neurophenomenology of volitional consciousness.

Contemplative neuroscience builds on neurophenomenology by proposing to supplement phenomenological reports from laypersons with reports from individu-

\footnotetext{
${ }^{1}$ These authors are careful to say minimally tainted rather than untainted. As phenomenologists such as Husserl, Heidegger, and Merleau-Ponty have discussed, commonsense understanding typically contains a large amount of philosophical sediment. Indeed, it has been claimed that our modern Western conception of the will is a philosophical idea invented by Augustine (e.g., Murphy 2006, p. 14).
} 
als who engage in the contemplative training of attention and meta-awareness (Lutz et al. 2007). Consider the Theravada Buddhist practice known as mindfulness of intention, often practiced during walking meditation. One cultivates an attention to and awareness of how the arising of an intention or volition precedes every movement. ${ }^{2}$ Usually intentions or volitions arise without this sort of awareness, and hence almost always lead automatically to action. When one notices their arising, however, one gains the ability to choose whether to act on them or not. One might think that this mental practice would interrupt the flow of action, but practitioners report that it actually helps one to reinhabit the flow of everyday action with heightened attunement and less mindless automaticity. This type of practice thus seems well suited for phenomenological investigations of the experience of volition and agency, and individuals accomplished in this practice might be able to provide information about conscious volitional processes unavailable to untrained individuals.

\section{Meditation and Neurodynamics}

From the perspective of dynamical neuroscience, transient conscious states are embodied in large-scale dynamical patterns of temporally coordinated neural activity across selective brain regions and areas (see Cosmelli et al. 2007 for a review). The voluntary generation of mental states in meditation seems to be no exception. In a recent study, Lutz and colleagues found distinct dynamical patterns of electrical brain activity recorded at the scalp in advanced Tibetan Buddhist meditators compared with novice practitioners during the voluntary generation of a specific kind of meditative state (Lutz et al. 2004). ${ }^{3}$ The brain waves of the longterm meditators showed high-amplitude gamma oscillations $(25-42 \mathrm{~Hz})$ and phase-

\footnotetext{
${ }^{2}$ The Sanskrit or Pali word cetana can be translated as either intention or volition. In Buddhist psychology cetana is one of the so-called constant mental factors that is present in every moment of consciousness and that functions to direct a mental state towards its object.

${ }^{3}$ The advanced meditators had undergone training for 10,000 to 50,000 hours over time periods ranging from 15 to 40 years. The novices had undergone training for 1 week before the data collection. The meditative state generated by the practitioners is known as nonreferential compassion. Tibetan Buddhists define compassion as the deep wish that others be happy and free from suffering. Usually this wish is directed towards a specific person or group, but nonreferential compassion, though necessarily other-directed, does not have a particular target. It is described as a state of being in which an unconditional feeling of loving-kindness and compassion pervades the whole mind, while awareness rests calmly and stably in complete openness without focusing on any particular object. This sheer awareness is considered to be invariant across all modes of consciousness regardless of the particular contents of consciousness. In nonreferential compassion meditation, the aim is to settle one's mind in this fundamental awareness while cultivating an intense feeling of compassion. This sort of "objectless meditation" is thus different from types of meditation that require concentration on an object (such as one's breath or a mental image). The cultivation of nonreferential compassion is thought to transform the mind in profound ways, by lessening fixation on self, counteracting afflictive states of mind (such as hatred and jealousy), creating a general sense of well-being and an unrestricted mental readiness and availability to help others, and counteracting mental dullness in meditation practice.
} 
synchrony over lateral frontoparietal electrodes during meditation. The ratio of gamma frequency activity to slow frequency activity $(4-13 \mathrm{~Hz})$ was higher in the baseline resting state before meditation for the adepts compared with the novices, and this ratio increased sharply during meditation and remained higher after meditation. In other words, brain activity in the resting state before meditation was already significantly different between the two groups, and this difference increased markedly during meditation. Furthermore, when the adepts entered the 30-second resting-state periods between the 60 -second meditation periods, their brain activity did not return to the initial premeditation baseline resting state, but instead displayed an ongoing baseline that reflected the previous meditation session.

These findings suggest that meditation induces short-term changes in neural activity and may bring about long-term changes in the brain. Nevertheless, this study cannot tell us whether contemplative training brings about these neural activity patterns or whether they reflect preexisting individual differences. A more recent longitudinal study, however, which examined the effects of three months of intensive training in insight meditation, showed that such training leads to increased control over the distribution of limited neural resources in attention (Slagter et al. 2007). ${ }^{4}$ This study provides direct evidence that systematic contemplative mental training affects the brain.

Another question concerns the significance of the high amplitude gamma oscillations seen during meditation (or the significance of gamma synchrony more generally in relation to conscious processes). Can such measures be related in any way to conscious experience?

To address this sort of question it would help to have more information about what is going on subjectively in people's minds from moment to moment as they engage some mental process. Working with highly trained contemplatives could be a real advantage here. These individuals spend years honing their capacities of attention, concentration, and meta-awareness, so it stands to reason they can describe their own subjective experience more precisely than can individuals who lack this kind of mental training.

Following this line of thought, Lutz and colleagues in a subsequent study (Lutz et al. 2006) asked long-term contemplatives to describe their subjective experience during meditation. Furthermore, instead of imposing already established terms from Western psychology, they asked the adept practitioners to report on their experiences using descriptive terms from their own tradition of contemplative theory and practice. Specifically, they asked the practitioners to report on the quality of "clarity" in their meditation.

\footnotetext{
${ }^{4}$ This study examined the effects of intensive meditation training on the attentional blink: When two targets are presented in close temporal proximity and at the same location in a sequence of visual stimuli, the second target is often not seen. This effect is thought to result from competition between the two targets for limited attentional resources. The study found that intensive meditation training resulted in a smaller attentional blink and reduced brain resource allocation to the first target, as reflected by a smaller P3b waveform (an event-related potential thought to index the allocation of attentional resources).
} 
Tibetan Buddhists use the term "clarity" to refer to the subjective intensity of the meditative state (see Lutz et al. 2007). Using the metaphor of light, they describe clarity as the luminosity or brilliance of the state. "Stability," on the other hand, shields the light or flame of clarity from flickering or going out. "Stability" refers both to the degree to which one stays in the meditative state, instead of being perturbed out of it, and the ease with which one regains the state if dislodged from it. Clarity and stability contrast respectively with dullness and excitation: A dull meditative state lacks clarity and an excited meditative state lacks stability. Thus a meditative state might be unstable - one gets repeatedly bumped out of the state but nonetheless clear or intensely experienced when one is in it. Or it could be stable but dull. In inexperienced meditators, clarity and stability tend to work against each other: The greater the stability, the more likely the meditative state is dull, the extreme being that one simply falls asleep; and the greater the intensity, the more likely one becomes excited and distracted, losing stability. The ideal meditative state finds a perfect balance between clarity and stability, so that neither dullness nor excitation impedes the mind.

When Lutz and colleagues asked the contemplative adepts to report on a scale from 1 to 9 any ongoing change in the clarity of their meditation (where 9 was defined as the peak of clarity they believed could be reached the day of the experiment), they found a strong correlation, over a time-course of several dozens of seconds, between self-reports of increasing clarity and the emergence of highamplitude gamma activity, particularly in frontal regions. Hence the gamma activity observed during this type of meditation seems closely related to the meditative state's phenomenal quality of clarity.

This way of using phenomenological information to help interpret neuroimaging data about conscious processes provides an example of the third working assumption of contemplative neuroscience mentioned at the beginning of this chapter and of the neurophenomenological approach more generally.

\section{Emergence}

The brain patterns seen in these and other neurodynamical studies of conscious processes are emergent in the following sense: They characterize the behavior of neural networks as complex (metastable) systems; they arise spontaneously given the local couplings among the network's components and the way those couplings are globally constrained and regulated; and they do not belong to any of the system's components taken singly or severally (Thompson \& Varela 2001).

Elsewhere I have argued that this sort of emergence may involve forms of nonseparability - the emergence of dynamic wholes that supersede or subsume their parts in irreducibly relational structures - and downward causation - the alteration of local behavior by global relational patterns (Thompson 2007).

I also argue, however, that the term "downward causation" is a misnomer. Complex-system causality is not a matter of a higher level acting downwards on a lower level. Rather, the whole entangled system moves at once and always as a 
result of both local interactions and the way the system's global organization shapes the local interactions (see Thompson 2007 for further discussion).

How might we conceptualize intention or volition in relation to this kind of complexity? Scott Kelso has proposed that an intention corresponds to an order parameter of the system's dynamics - a collective variable that constrains the system's behavior, either by stabilizing or destabilizing it (Kelso 1995, pp. 141-46). As examples he gives the influence of intention on the dynamics of bimanual coordination (e.g., in-phase and anti-phase finger-tapping) and the intentional perceptual reversal of ambiguous figures (Kelso 1995, pp. 218-25). In this connection it is worth noting that Oliva Carter and colleagues (Carter et al. 2005) found that long-term Tibetan Buddhist meditators can measurably alter normal fluctuations in conscious state induced by binocular rivalry (a kind of bistable perception). Specifically, complete perceptual stability for several minutes was induced by focused attention (one-pointed concentration) type meditation. Another example of the intentional modulation of dynamical neural activity is voluntarily affecting the course of an epileptic seizure by using cognitive countermeasures to prevent or interrupt it (Thompson \& Varela 2001; see also Le Van Quyen \& Petitmengin 2002; Petitmengin et al. 2006).

This model of the neurodynamics of intention may also be applicable to the voluntary generation of mental states in meditation. Although highly speculative, the general idea would be that contemplative mental training creates new types of global order parameters for the neural coordination dynamics underlying various conscious processes. The voluntary generation of mental states in meditation would thus correspond to inducing such order parameters in the brain.

\section{Volition as Intervention}

In this chapter I have taken a methodological approach and have not addressed the explanatory gap between consciousness and the brain or the philosophical problem of mental causation. Not much headway can be made on the first problem until we know more about both the phenomenology of conscious processes and the functioning of the brain as a complex system, and no progress is possible on the second problem if we do not get past the dichotomous concepts of the mental and the physical (inherited from Descartes) (Thompson 2007). For this reason, we need ways of conceptualizing psychological and biological causation that avoid these concepts. Here an "interventionist" account of "upward" and "downward" causation can help. ${ }^{5}$

According to the interventionist theory of causation, for $\mathrm{X}$ to be a cause of $\mathrm{Y}$ is for intervening on $\mathrm{X}$ to be a way of intervening on $\mathrm{Y}$. One way to intervene on biological events is to intervene on psychological events: Actively triggering a

\footnotetext{
${ }^{5}$ I owe this idea to the philosopher Michel Bitbol (2004). See Woodward (2003) for general discussion of the interventionist theory of causation, and Campbell (2007) for application to psychology.
} 
change in one's mental states by purely psychological means (contemplative mental training, emotion regulation, psychotherapy) may result in short-term and longterm changes to neural activity patterns, immune system function, hormonal patterns, and so on. And one way to intervene on psychological events is to intervene on biological events: Actively triggering a change in one's biological states by purely biobehavioral means (drugs, transcranial magnetic stimulation) may result in short-term and long-term changes to one's mental states. In this view, as Michel Bitbol remarks:

Making sense of upward and downward causation does not require a metaphysical distinction between the higher and basic levels of organization. Neither a substantial distinction, as in genuine dualism, nor a distinction between properties or structures as in the currently popular picture. It is enough to assume a duality of modes of access, or modes of intervention. If one intervenes at a higher level of organization, some effects of this action can then be detected by a mode of access specifically aimed at the lower level. This is downward causation. Conversely, if one intervenes at a microscopic level, some effects of this action can then be detected by a mode of access specifically aimed at a higher level of organization. This is upward causation. (Bitbol 2004)

This formulation allows us to say in a perfectly coherent way that contemplative experience acts downwardly on the brain by providing a distinct way of psychologically intervening on neurobiological processes.

\section{Conclusion}

Let me close with William James's observation that the essence of volition is effort of attention: "attention with effort is all that any case of volition implies. The essential achievement of the will, in short, when it is most 'voluntary,' is to attend to a difficult object and hold it fast before the mind" (James 1985, p. 317). For James, the question of psychological fact (rather than metaphysical speculation) in the free-will controversy "relates solely to the amount of effort of attention which we can at any time put forth. Are the duration and intensity of this effort fixed functions of the object, or are they not?" (p. 323). The line of thought pursued in this chapter suggests that sustained attention (which for advanced contemplatives may no longer be effortful), intention, and volition are not fixed functions of the object, but endogenously generated mental events that guide and control neural activities.

\section{References}

Bayne, T. (2006). Phenomenology and the feeling of doing: Wegner on the conscious will. In S. Pockett, W.P. Banks, and S. Gallagher (Eds.), Does consciousness cause behavior? An investigation of the nature of volition. Cambridge, MA: MIT Press/Bradford Books.

Bitbol, M. (2004). Downward causation: Concept and experience. Lecture presented at the conference De l'Autopoièse à la Neurophénomenologie/From Autopoiesis to Neurophenomenology: Un hommage à Francisco Varela/A Tribute to Francisco Varela. June 1820, 2004, Paris, France. 
Block, N. (1997). On a confusion about a function of consciousness. In N. Block, O. Flanagan, and G. Güzeldere (Eds.), The nature of consciousness: Philosophical debates (pp. 375-416). Cambridge, MA: MIT Press/A Bradford Book.

Campbell, J. (2007). An interventionist approach to causation in psychology. In A. Gopnik and L. Schulz (Eds.), Casual learning: Psychology, philosophy, and computation. New York: Oxford University Press.

Carter, O.L., Presti, D.E., Callistemon, C., Ungerer, Y., Lui, G.B., \& Pettigrew, J.D. (2005). Meditation alters perceptual rivalry in Tibetan Buddhist monks. Current Biology, 15, R412-13.

Cosmelli, D., Lachaux, J.-P., \& Thompson, E. (2007). Neurodynamical approaches to consciousness. In P.D. Zelazo, M. Moscovitch, and E. Thompson (Eds.), The Cambridge handbook of consciousness. New York: Cambridge University Press.

Dehaene, S., \& Naccache, L. (2001). Towards a cognitive neuroscience of consciousness: Basic evidence and a workspace framework. Cognition, 79, 1-37.

Hurlbert, R.T., \& Heavey, C.L. (2001). Telling what we know: Describing inner experience. Trends in Cognitive Sciences, 5, 400-403.

Jack, A.I., \& Roepstorff, A. (2002). Introspection and cognitive brain mapping: From stimulus-response to script-report. Trends in Cognitive Sciences, 6, 333-39.

James, W. (1985). Psychology: The briefer course. Notre Dame, IN: University of Notre Dame Press.

Kelso, J.A.S. (1995). Dynamic patterns: The self-organization of brain and behavior. Cambridge, MA: MIT Press/A Bradford Book.

Lambie, J.A., \& Marcel, A.J. (2002). Consciousness and the varieties of emotion experience: A theoretical framework. Psychological Review, 109, 219-59.

Le Van Quyen, M., \& Petitmengin, C. (2002). Neuronal dynamics and conscious experience: An example of reciprocal causation before epileptic seizures. Phenomenology and the Cognitive Sciences, 1, 169-80.

Lutz, A., \& Thompson, E. (2003). Neurophenomenology: Integrating subjective experience and brain dynamics in the neuroscience of consciousness. Journal of Consciousness Studies, 10, 31-52.

Lutz, A., Lachaux, J.-P., Martinerie, J., \& Varela, F.J. (2002). Guiding the study of brain dynamics by using first-person data: Synchrony patterns correlate with ongoing conscious states during a simple visual task. Proceedings of the National Academy of Sciences USA, 99, 1586-91.

Lutz, A., Greischar, L.L., Rawlings, N.B., Ricard, M., \& Davidson, R.J. (2004). Long-term meditators self-induce high-amplitude gamma synchrony during mental practice. Proceedings of the National Academy of Sciences USA, 101, 16369-73.

Lutz, A., Francis, A.D., \& Davidson, R.J. (2006). Changes in the tonic high-amplitude gamma oscillations during meditation correlate with long-term practitioners' verbal reports. Association for the Scientific Study of Consciousness Annual Meeting, poster presentation.

Lutz, A., Dunne, J.D., \& Davidson, R.J. (2007). Meditation and the neuroscience of consciousness: An introduction. In P.D. Zelazo, M. Moscovitch, and E. Thompson (Eds.), The Cambridge handbook of consciousness. New York: Cambridge University Press.

Lutz, A., Slagter, H., Dunne, J.D., \& Davidson, R.J. (2008). Attention regulation and monitoring in meditation. Trends in Cognitive Sciences, 12, 163-69.

Murphy, N. (2006). Bodies and souls, or spirited bodies? New York: Cambridge University Press.

Nahmias, E., Morris, S., Nadelhoffer, T., \& Turner, J. (2004). The phenomenology of free will. Journal of Consciousness Studies, 11, 162-79.

Nisbett, R.E., \& Wilson, T.D. (1977). Telling more than we can know: Verbal reports on mental processes. Psychological Review, 84, 231-59. 
Petitmengin, C. (2006). Describing one's subjective experience in the second person: An interview method for the science of consciousness. Phenomenology and the Cognitive Sciences, 5, 229-69.

Petitmengin, C., Baulac, M., \& Navarro, V. (2006). Seizure anticipation: Are neurophenomenological approaches able to detect preictal symptoms? Epilepsy and Behavior, 9, 298-306.

Slagter, H.A., Lutz, A., Greischar, L.L., Francis, A.D., Nieuwenhuis, S., Davis, J., \& Davidson, R.J. (2007). Mental training affects distribution of limited brain resources. PLoS Biology, 5/6, e138. http://www.plosbiology.org.

Schooler, J. (2002). Re-representing consciousness: Dissociations between experience and meta-consciousness. Trends in Cognitive Sciences, 6, 339-44.

Schooler, J., \& Schreiber, C.A. (2004). Experience, meta-consciousness, and the paradox of introspection. Journal of Consciousness Studies, 11, 17-39.

Thompson, E. (2007). Mind in life: Biology, phenomenology, and the sciences of mind. Cambridge, MA: Harvard University Press.

Thompson, E., \& Varela, F.J. (2001). Radical embodiment: Neural dynamics and consciousness. Trends in Cognitive Sciences, 5, 418-25.

Varela, F.J. (1996). Neurophenomenology: A methodological remedy for the hard problem. Journal of Consciousness Studies, 3, 330-50.

Wegner, D.M. (2002). The illusion of conscious will. Cambridge, MA: MIT Press/Bradford Books.

Wegner, D.M. (2004). Précis of The illusion of conscious will. Behavioral and Brain Sciences, 27, 649-92.

Woodward, J. (2003). Making things happen: A theory of causal explanation. New York: Oxford University Press. 